\title{
A Quantitative Comparison of Edges in 3D Intraoperative Ultrasound and Preoperative MR Images of the Brain
}

\author{
Karen E. Lunn ${ }^{1}$, Alex Hartov ${ }^{1,2,3}$, Eric W. Hansen ${ }^{1}$, Hai Sun ${ }^{1}$, \\ David W. Roberts ${ }^{2,3}$, and Keith D. Paulsen ${ }^{1,2,3}$ \\ ${ }^{1}$ Thayer School of Engineering, Dartmouth College, Hanover, NH 03755 USA \\ ${ }^{2}$ Dartmouth Hitchcock Medical Center, Lebanon, NH 03766 USA \\ ${ }^{3}$ Norris Cotton Cancer Center, Lebanon, NH 03766 USA
}

\begin{abstract}
The displacement of brain tissue during neurosurgery is a significant source of error for image-guidance systems. We are investigating the use of a computational model to predict brain shift and warp preoperative MR images accordingly. 3D ultrasound appears to be a valid means of acquiring intraoperative images to use as sparse data for guiding the model calculations. We present here a study of edge detection in MR and ultrasound images to investigate the accuracy of our ultrasound system and explore methods of extracting sparse data. Ultrasound images are acquired prior to deformation and compared to their corresponding oblique MR/CT images. Results from phantom images show misalignment by an average of $2.2 \pm 1.67$ degrees, $0.90 \pm 0.82 \mathrm{~mm}$ in $\mathrm{x}$, and $0.87 \pm 0.87 \mathrm{~mm}$ in $\mathrm{y}$. Patient images are misaligned by an average of $2.2 \pm 1.4$ degrees, $1.24 \pm 0.89 \mathrm{~mm}$ in $\mathrm{x}$, and $0.97 \pm 0.87 \mathrm{~mm}$ in $\mathrm{y}$.
\end{abstract}

\section{Introduction}

The introduction of image-guidance systems has been a significant advancement in the field of neurosurgery, giving surgeons a powerful navigational tool and an increased level of accuracy. MR images of the patient are acquired prior to surgery, and registered to the patient in the operating room. Surgical tools can then be tracked and related to the MR images through coordinate transformations obtained from the registration procedure. This system provides feedback to the surgeon to help more accurately place surgical tools and navigate through the brain.

The accuracy of an image-guidance system depends on the assumption that registration involves a rigid transformation of the patient's brain in the operating room (OR) to the preoperative image volume. A number of studies have shown, however, that the brain shifts with respect to the skull, thus making the assumption of a rigid transformation invalid [1-4]. Strategies for reducing this source of error are being explored, with intraoperative MR, brain deformation modeling, and intraoperative ultrasound, or some combination of the three, emerging as the most common solutions. [5-10]. While MR has the advantage of being high contrast with high signal to noise ratio, it also has the drawbacks of being expensive, time consuming, and bulky. Ultrasound images can also provide intraoperative updates of the brain during surgery, and has the advantages of being real-time, portable, and far less expensive. However, due to the low signal to noise ratio and speckle 
characteristic of ultrasound data, a full volume representation of brain deformation would be difficult to construct from ultrasound alone. In contrast, a computational model could retain the advantages of MR image quality by warping preoperative images based on computed displacements. In previous studies, we have demonstrated the feasibility of using a model based on consolidation physics to recover brain shift [11-13]. Nonetheless, it is likely critical to obtain sparse intraoperative data to help drive model calculations. The advantages of ultrasound make it well suited for providing this information.

This paper describes an initial study designed to validate the use of 3D ultrasound as sparse data. We outline our procedure for relating the ultrasound image to the MR stack and matching homologous features. As a measure of error, we present the degree of rotation and translation needed to align each pair of images. Finally, we explore the results of edge detection in MR and ultrasound in the context of extracting sparse displacement data. Specifically, we investigate the level of feature congruence that may be obtainable in the OR during clinical cases that lack high contrast structures (e.g. tumor margins) or well-demarked surfaces (e.g. ventricles) that may not be present or visible in many cases.

\section{Method}

The initial registration of ultrasound and MR data is performed using an optical tracking system, which records the location of the ultrasound scanhead in real time. In this stage of registration, each ultrasound image is registered to the MR image stack, making it possible to reconstruct an oblique MR slice in the same orientation as the ultrasound image plane. The registration is then refined locally by aligning the edges of corresponding features in ultrasound and MR data. This image-based reregistration is classical in nature, and still in preliminary stages of development. Others groups have shown success in using mutual information (MI) or correlation ratio (CR) to co-register ultrasound and MR images (or other multimodal data) [1417]. These image-based methods seek to register ultrasound and MR without the aid of tracking systems, and therefore the registration is performed over a larger volume. Our goal is somewhat different; we seek to improve the initial tracking-based registration over a localized region of interest, by exploiting some operator input, in order to investigate the visibility of less obvious feature edges in both ultrasound and MR. As a result, we opted to use the relatively simple techniques described here, rather than more sophisticated image-based registration strategies despite the fact that future work may benefit from incorporation of MI or CR into this refinement process.

\subsection{Materials}

Our 3D ultrasound system consists of a 5MHZ intraoperative ultrasonography system (Aloka Model 633; Corometrix Medical Systems, Wallingford, CT), a computer equipped with a frame grabber (model DT3155; Data Translation, Marlboro, MA), and a 3D optical tracker (Polaris; Northern Digital, Waterloo, Ontario, CAN). Image processing was performed using intrinsic functions available in Matlab (Version 6.1, Mathworks), as well as custom-built software, also written in Matlab. 


\subsection{MR Reconstruction}

Points in ultrasound coordinates are mapped to MR coordinates through a series of coordinate transformations. These successive mappings are summarized in Eq.1,

$$
P_{m r}={ }^{m r} T_{w}{ }^{w} T_{t r}{ }^{t r} T_{u s} P_{u s},
$$

where $P_{m r}$ is a vector containing points in MR coordinates, $P_{u s}$ is the corresponding vector for points in ultrasound coordinates, and the remaining terms are the three transformation matrices: the transformation from ultrasound to tracker coordinates $\left.{ }^{t r} T_{u s}\right)$, which is obtained through a complex calibration procedure; the transformation from tracker coordinates to world coordinates $\left({ }^{w} T_{t r}\right)$, which is provided by the Polaris optical tracking system; and the transformation from world coordinates to MR coordinates $\left({ }^{m r} T_{w}\right)$, which is obtained through patient registration. Previous analysis of our calibration procedure has shown that by mapping the ultrasound calibration points to world coordinates, we can recover the original world coordinates to within an average RMS of $1.5 \mathrm{~mm}$. Furthermore, an independent test of our ability to map a point in ultrasound to its world coordinates showed that we could do so with an average error of $2.32 \mathrm{~mm}$. [19]. Our Polaris optical tracker has been found to have an accuracy of approximately $0.33 \mathrm{~mm}$, which is comparable to results reported by other groups $[5,20]$.

Reconstructing an oblique MR image begins by mapping each pixel in an ultrasound image to the MR coordinate system, using Eq.1. The corresponding MR pixel is then obtained by finding the nearest voxel. This is completed for every pixel in the ultrasound image, resulting in two images of the same resolution $(480 \times 640$ pixels). With an accurate registration, this pair of images should depict the same features, though they may appear different in each modality. As a qualitative approach to assessing alignment, we also create a colored composite of the two images superimposed, with MR in the red channel, and ultrasound in the green channel. This helps to visually identify common features in the two images.

\subsection{Image Processing}

We assess the accuracy of the MR reconstruction by extracting homologous features from the two images using classical methods, which are mostly manual. Before running an edge detection algorithm, the images are preprocessed to reduce noise and enhance edge features. The ultrasound images are first are smoothed and enhanced using a mean (averaging filter), followed by a median filter. For an $n \times n$ kernel, the mean filter calculates the average of the pixels in the neighborhood, and reassigns the mean intensity to the center pixel. This has the effect of blurring the image, with a larger kernel creating greater blurring. The median filter calculates the median of its $n$ $\times \mathrm{n}$ neighborhood, and assigns that value to the center pixel. MR images are enhanced using an anisotropic diffusion algorithm [21]. This filter is designed to smooth homogenous regions, while retaining sharp edges. The level of smoothness imposed can be increased or decreased by changing the level of iterations, as well as the degree of diffusion. For more challenging images, sometimes a combination of median, mean and anisotropic diffusion filters were used on both ultrasound and MR images. A 
custom designed graphical user interface allows the user to interactively change the size of the median and mean filters, as well as the iterations and diffusion level, to enhance features that are recognizable in both ultrasound and MR.

Following preprocessing, the edges in the images are identified using classical edge detection algorithms. For ultrasound images, edges are defined as the zero crossings of the Laplacian of a Gaussian filter. For MR images, we search for the maximum of the gradient, employing the Sobel approximation calculate the magnitude of the gradient. The threshold of these operators can be interactively changed to produce fewer or more edges. These operations are prepackaged functions in the Matlab Image Processing Toolbox. Edge patterns that appear in both images are selected. Selected edges are dilated to assist the edge matching algorithm in searching for common edges.

To match edges, the user must initialize the alignment by identifying a matching feature in the ultrasound and MR images. The algorithm then automatically searches for the proper alignment of the edges, by searching \pm 5 units in rotation and translation for the combination of these parameters that results in a minimum value in the absolute difference between the two binary images. This method was tested on a simple simulation, where a binary image of a triangle edge was rotated and translated by a known amount, and those parameters were recovered using the algorithm described above. For all trials of initialization, the automatic search proved to capture the rotation and translation better than a manual approach. The original transformation was recovered with exact matching in rotation, and to within 0.3 pixels in $\mathrm{x}$ and $\mathrm{y}$ translation.

\section{Results}

\subsection{Phantom}

This edge matching procedure was performed on both phantom images and clinical data. The phantom consisted of a Plexiglas water tank strung with wires. These features were displayed clearly and with bright contrast in both ultrasound and CT images. As a result, edge detection and edge matching was a simple process. Figure 1A. shows a sample image pair, with the walls and wires of the tank clearly visible in both modalities. Before processing, both images are cropped to the same size to limit the computation to the region of interest. The ultrasound image of the phantom was filtered using a $5 \times 5$ mean filter, followed by a $15 \times 15$ median filter. The CT image of the phantom was filtered using an anisotropic filter, with 15 iterations and a diffusion level of 10. The resulting edges, after deleting artifacts, are displayed in the last row. For the image shown in figure $1 \mathrm{~A}$, the $\mathrm{CT}$ image was rotated by 0 degrees, and translated approximately $.34 \mathrm{~mm}$ (2.5 pixels) in $\mathrm{x}$ and $0.69 \mathrm{~mm}$ (5 pixels) in $\mathrm{y}$ to match with the ultrasound image. For the remaining 34 images, using four different ultrasound scale settings, images were rotated by an average of 2.2 degrees, and translated by $0.89 \mathrm{~mm}$ in $\mathrm{x}$ and $0.89 \mathrm{~mm}$ in $\mathrm{y}$, to match the edges. Table 1 presents the results obtained for each scale. 
Table 1. Phantom results. Shows rotation and translation necessary to align US and CT images.

\begin{tabular}{|l|c|c|c|}
\hline depth scale & rotation (degrees) & x translation $(\mathrm{mm})$ & y translation $(\mathrm{mm})$ \\
\hline $6 \mathrm{~cm}$ & $1.91 \pm 1.51$ & $0.65 \pm 0.39$ & $0.59 \pm 0.25$ \\
\hline $8 \mathrm{~cm}$ & $2.18 \pm 2.09$ & $0.83 \pm 0.48$ & $0.50 \pm 0.34$ \\
\hline $12 \mathrm{~cm}$ & $2.10 \pm 1.37$ & $1.02 \pm 1.00$ & $0.69 \pm 0.57$ \\
\hline $16 \mathrm{~cm}$ & $2.77 \pm 1.69$ & $1.07 \pm 1.12$ & $1.57 \pm 1.29$ \\
\hline
\end{tabular}

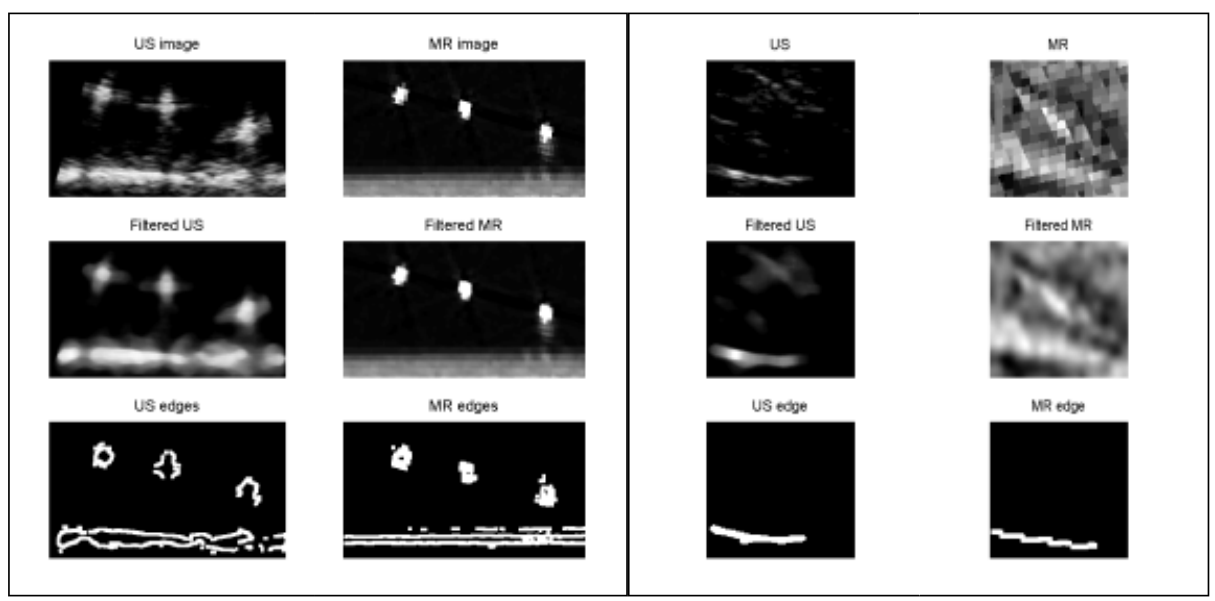

Figure 1. A. Images on left show ultrasound (far left) and CT (middle left) images of phantom tank, filtered images, and detected edges used to align images. B. Images on right show ultrasound (middle right) and MR (far right) of section of patient's brain. Below original image, filtered images and detected edges are displayed.

\subsection{Patient}

This combination of preprocessing, selection of edges, and semi-automatic alignment was also performed for 18 images of one patient. All of these images were obtained after the craniotomy, before the dura was removed. Therefore, we can assume that little or no deformation has occurred. Figure $1 \mathrm{~b}$ illustrates a typical example from this case, where the white to gray matter boundary displayed in MR corresponds to a bright line in ultrasound. For this image set, the ultrasound image was preprocessed with a $5 \times 5$ mean filter, followed by a $15 \times 15$ median filter. The MR image was smoothed using an anisotropic diffusion filter, with 30 iterations and diffusion level 30. These images were matched with approximately 2 degrees rotation, $0.8 \mathrm{~mm}(6$ pixels) in $\mathrm{x}$, and $1.4 \mathrm{~mm}$ (10.2 pixels) in $\mathrm{y}$. A more difficult case is displayed in figure 2. The images show similar, but less exact patterns. In this case, the both pairs of images were first filtered with mean and median filters to blur the edges and reduce noise. Next, they were filtered using the anisotropic diffusion algorithm to further blend homogenous regions while retaining sharp edges. The smoothed images are 
displayed in the middle row of figure 2. The last row depicts the resulting edges from these filtered images, overlaid on the original images. This particular image set was determined to be misaligned by -3 degrees, $1.3 \mathrm{~mm}$ (9.5 pixels) in $\mathrm{x}$, and $1.6 \mathrm{~mm}$ (11.7 pixels) in y. The results of all of the images show that they are properly aligned by rotating by an average of $2.2 \pm 1.4$ degrees and translating by an average of $1.24 \pm$ $0.89 \mathrm{~mm}$ in $\mathrm{x}$ and $0.97 \pm 0.87 \mathrm{~mm}$ in $\mathrm{y}$.

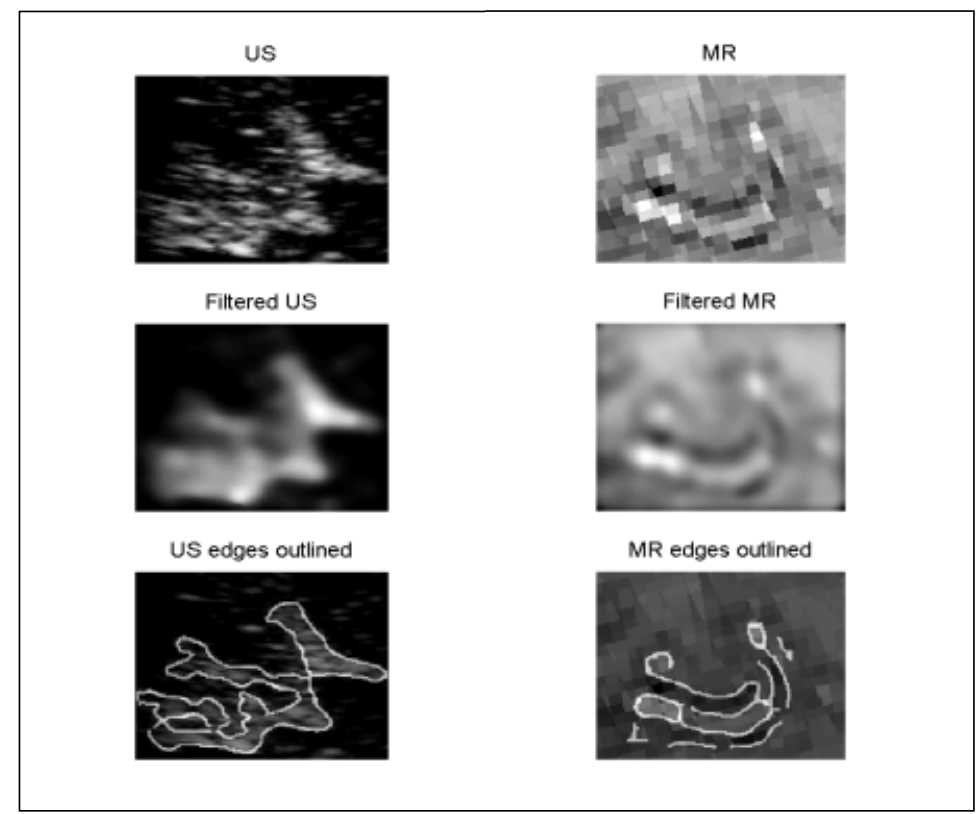

Figure 2. Ultrasound (left) and MR images of patient brain, with filtered and edge detected results. Images represent a region of approximately 1.5 by $2.0 \mathrm{~cm}$.

\section{Discussion and Conclusions}

The results of these studies validate the accuracy of our 3D ultrasound system and provide some insight into possible methods of extracting quantitative sparse data from intraoperative ultrasound images. The level of accuracy achieved in matching the phantom images represent a significant achievement in our goal of using intraoperative ultrasound in conjunction with a brain deformation model. The phantom provided an excellent set of test images, as its features produced clear, high contrast images of its walls and wires. The clarity of edge contours in both ultrasound and CT left little ambiguity in choosing the correct features to match. Therefore, we have confidence in our conclusion that for a given ultrasound image, we can properly reconstruct the corresponding oblique MR image to within approximately $1 \mathrm{~mm}$. 
It would be convenient if MR and ultrasound edges were always as apparent as they are in the phantom; unfortunately, this is not usually the case. Images of brain features such as the falx, tumors, and ventricles, which can be high contrast and easy to recognize, have been used in other studies to register images or characterize deformation $[6,16,22]$. However, these features are not always present in the region of interest. With the patient data from this study, we show that it is possible to match more subtle brain features, such as the interface between white and gray matter, as well as sulcal patterns. Furthermore, we propose that when similar patterns emerge in both images, these could be used for matching, even if the exact nature of the feature is unclear.

The results of the phantom study lead us to conclude that edges which appear to line up in the MR and ultrasound image do, in fact, represent the same features. For example, the distinction between white and gray matter is readily apparent in MR images. By superimposing the ultrasound images on their oblique MR counterparts, it was clear that strong ultrasonic reflections were produced at the white/gray matter interface. Figure 2 shows a typical example of this echogenicity. Note that the bright line in the ultrasound image is located in the same region as the gray/white matter boundary displayed in the MR image.
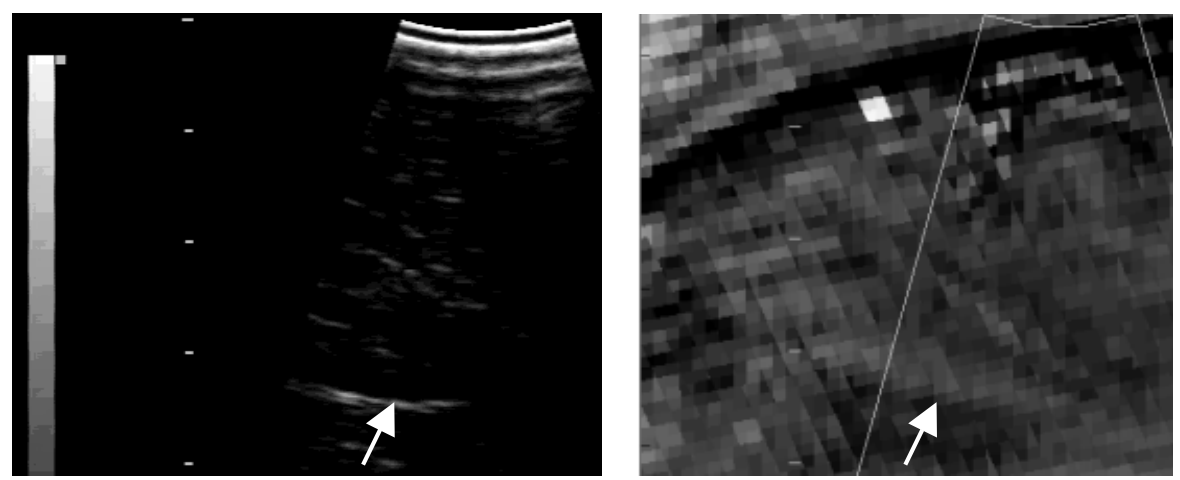

Figure 3. Cropped ultrasound image (left) and its corresponding oblique MR reconstruction (right). Arrow points to white/gray matter interface in both images. For reference, the ultrasound scale and wedge shape are overlayed on the MR image

By searching within a region of interest in the two images, we were also able to match edges of more complex features with correlating patterns. For example, the images shown in Figure 3 show similar patterns of parallel horizontal edges in the lower left corner, and diagonal edges in the center. In this case, the features appear to be sulcal patterns. It is important to note, however, that the features do not need to be correctly categorized in order to recognize patterns in edges and find the translation and rotation necessary to align the two sets of edges. Furthermore, while these features were already closely aligned, it seems feasible to use this method of pattern searching in ultrasound images depicting deformed brains, where features have moved from their preoperative location. The region of interest would have to be greater in order to find patterns in the displaced tissue that match patterns in the MR. 
After identifying similar features, the rotation and translation could be calculated using a similar edge matching technique. Finally, the results of this displacement calculation would be used as inputs to the computational model. Obviously, this strategy needs to be explored further to validate its usefulness in the deformed case. It may be that more sophisticated, automatic, image-based registration methods, such as MI or CR, will need to be employed in order to realize a robust global edge-matching technique.

The results of the patient case should be interpreted with some caution. The images analyzed represent the best ultrasound/MR pairs, in the sense that the features present in one image are clearly related to those in the second image. Nonetheless, the results are valuable in that they demonstrate the level of accuracy that can be achieved. Furthermore, this selective process is a practical strategy for using sparse ultrasound data in conjunction with the brain model. That is, it seems reasonable to use only the best ultrasound/MR pairs to calculate local displacement as input to the model. Since our motivation for using intraoperative ultrasound is to gain sparse displacement data, rather than a full volume description, this limited selection is compatible with our strategy.

Another possible drawback of the method described here is that while we have three-dimensional data, the edge matching analysis is performed in two dimensions. Although this simplification is acceptable for an initial study, the displacement calculated would represent only the projection of the actual displacement onto the $2 \mathrm{D}$ image plane. It has been pointed out that if the $2 \mathrm{D}$ image lines up with the principle direction of displacement, the $2 \mathrm{D}$ image may be adequate [6]. We have begun to investigate the use of $3 \mathrm{D}$ data, with some encouraging initial results. Our current approach still uses the 2D ultrasound image plane, but searches for a better match to an oblique MR plane by integrating the 3D MR image volume. Figure 4 demonstrates the results from matching one ultrasound image with an improved oblique MR slice. This 3D method warrants further development; it does not yet incorporate multiple ultrasound images in $3 \mathrm{D}$, which may prove to be more robust. More sophisticated image processing techniques also need to be developed in order to attain our goal of predicting shift in real time.
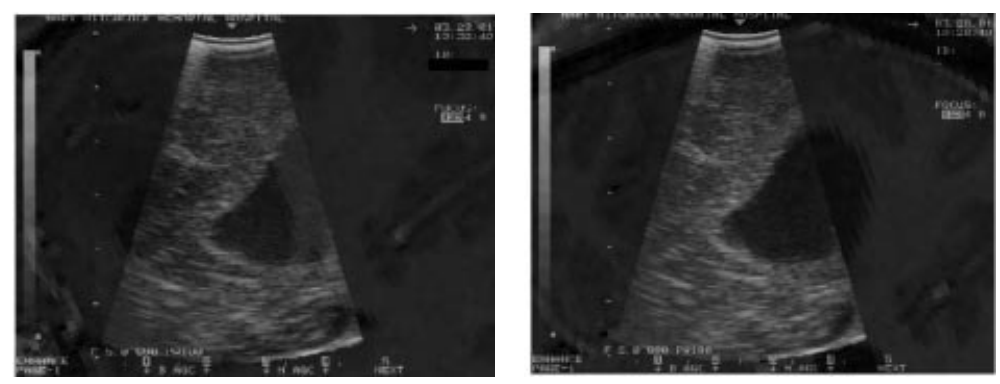

Figure 4. Results from 3D edge matching on one set of ultrasound and MR images. Left figure displays overlap of images before re-registration. Right figure displays images after 3D edge matching isperformed. 
Finally, we have proposed that less obvious features, such as gray/white matter interfaces and sulcal patterns are potentially useful for extracting sparse intraoperative data on brain tissue motion. While we present some initial findings on the relative appearance of these structures in ultrasound and MR, a more extensive comparison of the characteristics of brain features in various modalities would be quite helpful. Such a study could provide a priori knowledge concerning feature boundaries, which would be useful in automating the steps of segmentation and edge matching.

The underlying assumption in the work presented here is that the features occurring in both image sets do in fact correspond to the same physical structures Given this premise questions also arise as to whether the simple image processing and feature extraction techniques we used apply appropriately across the differing image scales of the two modalities. Certainly, the results from the phantom study (Fig. 1) and high contrast in vivo data (Fig. 4) which exploited the same techniques suggest they do. However, further study is required to thoroughly substantiate the approach in more subtle low contrast cases (e.g. Fig. 2,3). Our intent was not to definitively validate the methodology described herein, or even to necessarily endorse the approach above other possibilities, but rather to indicate that opportunities exist for localized lowcontrast feature matching between intraoperative US and preoperative MR that could constitute sparse data for full-volume MR updates achieved through computational modeling.

\section{References}

1. D.W. Roberts, A. Hartov, F.E. Kennedy, M.I. Miga, K.D. Paulsen: Intraoperative Brain Shift and Deformation: A Quantitative Analysis of Cortical Displacement in 28 Cases. Neurosurgery, vol.43: 49-760, 1998.

2. N. Hata, A. Nabavi, W.M. Wells, S.K. Warfield, R. Kikinis, P.M. Black, F.A. Jolesz: Three-dimensional optical flow method for measurement of volumetric brain deformation from intraoperative MR images. Journal of Computer Assisted Tomography, vol.24(4): 531-8, 2000.

3. C.R. Maurer, D.L. Hill, A.J. Martin, H. Liu, M. McCue, D. Rueckert, D. Lloret, W.A. Hall, R.E. Maxwell, D.J. Hawkes, C.L. Truwit: Investigation of intraoperative brain deformation using a 1.5-T interventional MR system: preliminary results. IEEE Transactions on Medical Imaging, vol.17(5): 817-25, 1998.

4. D.L. Hill, C.R. Maurer, R.J. Maciunas, J.A. Barwise, J.M. Fitzpatrick, M.Y. Wang: Measurement of intraoperative brain surface deformation under a craniotomy. Neurosurgery, vol.43(3): 514-26, 1998.

5. D.G. Gobbi, R.M. Comeau, and T.M. Peters: Ultrasound Probe Tracking for Real-Time Ultrasound/MRI Overlay and Visualization of Brain Shift. Medical image computing and computer-assisted intervention--MICCAI'99 : second international conference : 920-927, 1999.

6. R.M. Comeau, A. Fenster, T.M. Peters: Intraoperative US in Interactive Image-guided Neurosurgery. RadioGraphics, vol. 18(4): 1019-1027, 1998.

7. A. Jödicke, W. Deinsberger, H. Erbe, A. Kriete, D.K. Böker: Intraoperative ThreeDimensional Ultrasonography: An Approach to Register Brain Shift using Multidimensional Image Processing. Minim. Invas. Neurosurg 41: 13-19, 1998.

8. D.G. Gobbi, R.M. Comeau, T.M. Peters: Ultrasound/MRI Overlay with Image Warping For Neurosurgery. Medical image computing and computer-assisted intervention-MICCAI 2000: 106-114, 2000. 
9. D.W. Roberts, M.I. Miga, A. Hartov, S. Eisner, J.M. Lemery, F.E. Kennedy, K.D. Paulsen: Intraoperatively Updated Neuroimaging Using Brain Modeling and Sparse Data. Neurosurgery, vol.45(5): 1199-1207, 1999.

10. C. Nimsky, O. Ganslandt, S. Cerny, P. Hastreiter, G. Greiner, R. Fahlbusch: Quantification of, Visualization of, and Compensation for Brain Shift Using Intraoperative Magnetic Resonance Imaging. Neurosurgery, vol.47(5): 1070-1080, 2000.

11. M.I. Miga, K.D. Paulsen, P.J. Hoopes, F.E. Kennedy, A. Hartov, D.W. Roberts: In Vivo Quantification of a Homogeneous Brain Deformation Model for Updating Preoperative Images During Surgery. IEEE Transactions on Biomedical Engineering. vol. 47(2): $266-$ 273, 2000.

12. K.P. Paulsen, M.I. Miga, F.E. Kennedy, P.J. Hoopes, A. Hartov, D.W. Roberts: A Computational Model for Tracking Subseruface Tissue Deformation During Stereotactic Neurosurgery. IEEE Transactions on Biomedical Engineering, vol.46(2): 213-225, 1999.

13. L. Platenik: Investigation of Retraction Deformation Modeling for Model-Updated ImageGuided Stereotactic Neurosurgery. M.S. Thesis, Thayer School of Engineering, Dartmouth College, January 2001.

14. J.M. Blackall, D. Ruechert, C.R. Maurer, G.P. Penney, G.L.G. Hill, and D.J. Hawkes. An Image Registration Approach to Automated Calibration for Freehand 3D Ultrasound. Medical image computing and computer-assisted intervention--MICCAI 2000: 462-471, 2000.

15. A. Roche, G. Malandain, and N. Ayache. Unifying Maximum Likelihood Approaches in Medical Image Registration. International Journal of Imaging Systems and Technology, vol.11, (2000) 71-80

16. A. Roche, X. Pennec, M. Rudolf, D.P. Auer, G. Malandain, S. Ourselin, L.M. Auer, and N. Ayache. Generalized Correlation Ratio for Rigid Registration of 3D Ultrasound with MR Images. Medical image computing and computer-assisted intervention--MICCAI 2000: (2000) 567-577

17. J.P.W. Pluim, J.B.A. Maintz, and M.A. Viergever. Image Registration by Maximization of Combined Mutual Information and Gradient Information. Medical image computing and computer-assisted intervention--MICCAI 2000: (2000) 452-461

18. A. Hartov, S.D. Eisner, D.W. Roberts, K.D. Paulsen, L.A. Platenik, M.I. Miga: Error analysis for a free-hand three-dimensional ultrasound system for neuronavigation. Neurosurgical Focus, vol.6(3), 1999. <http://www.neurosurgery.org/focus/mar99/6-35.html>

19. K.E. Lunn, A. Hartov, F.E. Kennedy, M.I. Miga, D.W. Roberts, L.A. Platenik, K.P. Paulsen: 3D Ultrasound as Sparse Data for Intraoperative Brain Deformation Model. In Proceedings of SPIE--The International Society for Optical Engineering. Medical Imaging 2001: Ultrasonic Imaging and Signal Processing.

20. Northern Digital, Inc. Products: POLARIS, <http://www.ndigital.com/polaris.html $>$, $12 / 01 / 00$

21. P. Perona, J. Malik: Scale-Space and Edge Detection Using Anisotropic Diffusion. IEEE Transactions on Pattern Analysis and Machine Intelligence. vol.12(7): 629-639, 1990.

22. H. Erbe, A. Kriete, A. Jödicke, W. Deinsberger, D.K. Böker: 3D-Ultrasonography and Imaging Matching for Detection of Brain Shift During Intracranial Surgery. Computer Assisted Radiology: Proceedings of the International Symposium on Computer and Communication Systems for Image Guided Diagnosis and Therapy: 225-230, 1996. 